\title{
ANALYSIS OF NEURONAL FUNCTIONS BASED ON FEYNMAN DIAGRAMS
}

\author{
J.A. Martin-Pereda <jamp@tfo.upm.es>, A.P. Gonzalez-Marcos \\ ETS Ingenieros de Telecomunicacion \\ Universidad Politecnica de Madrid \\ 28040 MADRID. SPAIN
}

Abstract - A method to study some neuronal functions, based on the use of the Feynman diagrams, employed in Many-Body Theory, is reported. An equation obtained from the neuron cable theory is the basis for the method. The Green function for this equation is obtained under some simple boundary conditions. An excitatory signal, with different conditions concerning high and pulse duration, is employed as input signal. Different responses have been obtained.

Key words: neural functions, neuron modelling, neuronal behaviour.

\section{INTRODUCTION}

Several theories have been employed in the last years concerning the modelling of some functions of the neuronal system. These models go from the cable theory to more sophisticated theories covering a large variety of parameters and behaviours. Everyone of these models employs one of the two possible approaches: analytical, with solutions obtained from mathematical solutions or numerical, with computer based solutions.

A new approach is adopted in this paper. The basis was the use of a technique used in Many Body Theory. An extension of a previous work [1] is reported in this paper.

\section{BASIS OF THE METHOD AND RESULTS}

As it is well known, one of the possible approaches to study signal propagation in neuronal systems is the use of the cable equation. One of the well-known possible formulations for this equation is [2]

$$
\lambda^{2} \frac{\partial^{2} E}{\partial x^{2}}=\tau \frac{\partial E}{\partial t}+V
$$

A solution can be obtained from its Green's function. The advantage of this way to work is that Green's function corresponds to the propagator of the system. And this propagator may be handled with techniques employed in Many Body Systems [3]. Their Feynman diagrams give an straightforward pictorial representation from where many of the most important properties can be obtained. In the present case, one of the analysed cases is the represented by this diagram.

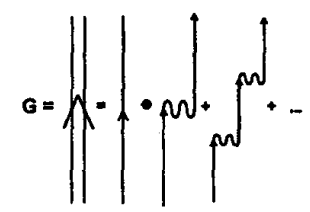

This situation corresponds to the propagation of a certain stimulus $\mathrm{V}$ along a neuronal network. This propagation, depicted by the double line with an arrow, may be decomposed into the different ways the signal may propagate along the system. These ways are, for instance, a direct propagation (simple line), plus propagation by means of two interacting neurones, plus propagation by three interacting neurones and so on. This pictorial representation corresponds to

$$
G(w)=G_{o}\left[1+i G_{o}(-i V)+\left(i G_{o}\right)^{2}(-i V)^{2}+. .\right]=\frac{G_{o}}{1-G_{o} V}
$$

where $G(w)$ stands for the Green's function of the perturbed system and $G_{0}$ for the unperturbed one. The analyzed case is when

$$
G_{o}(t)=-i\left[\Theta_{t} e^{-i w_{0} t}+\Theta_{-t} e^{i w_{o} t}\right]
$$

$w_{0}$ is the neurone natural frequency. Hence, the Fourier transform of $\mathrm{G}$ will be

$$
G(w)=\frac{1}{\left(w-w_{0}\right)-V}
$$

A possible situation to study is the excitation by a pulse of amplitude $\mathrm{A}$ and time duration $\mathrm{T}$. If $V=A$ for $|\mathrm{t}|<\mathrm{T} / 2$ and $V=0$ for $|\mathrm{t}|>\mathrm{T} / 2$, hence

$$
\begin{gathered}
V(w)=A T \frac{\sin (w T / 2)}{w T / 2} \text { and so } \\
G(w)=\frac{1}{\left(w-w_{o}\right)-A T(\sin (w T / 2)) /(w T / 2)}
\end{gathered}
$$

Several situations have been analysed corresponding to different values for $\mathrm{T}$ and $\mathrm{A}$. A value of $174.6 \mathrm{~Hz}$ for the natural frequency (squid giant axons case) was taken. Two solutions appear at the Fig. 1.a and 1.b. Fig 1.a correspond to $A=0.5$ and Fig. 1.b to $A=100$.
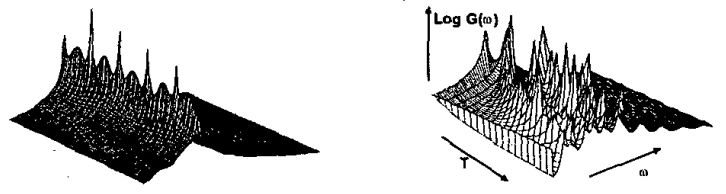

Fig. 1

It is clear from these results, that the stimulus characteristics determine the size of the generator potential and the frequency content.

\section{REFERENCES}

[1] J.A. Martín-Pereda \& A. González-Marcos, SPIE, 2036, 162-173 (1993).

[2] G.M. Shepherd, "The Synaptic Organization of the Brain". Oxford. 1990.

[3] R.D. Mattuck, "A Guide to Feynman Diagrams in the Many-Body Problem”. Dover. 1992. 